\title{
A note on Purcell's basic explanation of magnetic forces
}

\author{
Germano D'Abramo \\ Ministero dell'Istruzione, dell'Università e della Ricerca, 00041, Albano Laziale, RM, \\ Italy \\ E-mail: germano.dabramo@gmail.com
}

Dicember 21, 2019

\begin{abstract}
In the 1960s, E.M. Purcell presented a basic explanation of the magnetic force experienced by a test charge moving parallel to a stationary current-carrying wire. According to Purcell's derivation, this force results from the difference between the relativistic length contraction of the distance among the stationary positive charges of the wire and the relativistic length contraction of the distance among the negative charges moving in the wire, when the charges are observed in the rest frame of the test charge. The contraction difference generates a charge density unbalance that in the rest frame of the test charge is experienced as an electrostatic force, while in the lab frame is perceived as the magnetic force. In the present paper, we show that Purcell's approach is problematic since it generates inconsistencies and paradoxes. We maintain that Purcell's derivation has only an illustrative and expository value and should not be taken literally as describing something that really and physically happens in the wire. Furthermore, we believe that the difficulties pointed out here should be explicitly presented and discussed when introducing Purcell's approach in physics courses at the undergraduate and graduate levels.
\end{abstract}

Keywords: electromagnetism, Lorentz force, Ampère force law, special relativity, length contraction, Purcell's basic explanation of magnetic forces

Submitted to: Eur. J. Phys.

\section{Introduction}

The magnetic force felt by a charge moving parallel to a fixed current-carrying wire is in fact an electrostatic force when it is observed in the rest frame of the charge. E.M. Purcell provided a basic explanation of this occurrence [1]: in the rest frame of the moving charge, the lattice positive charges of the wire are seen as moving and then their 
density is modified by Lorentz contraction differently than the density of the moving electrons which constitute the current. This charge unbalance is allegedly at the origin of the magnetic force (i.e. the Lorentz force).

We now explicitly derive Purcell's result referring to the classical derivations that can be found in the literature [1, 2, 3, 4]. Consider a charge $q$ moving with velocity $v_{0}$, close by and parallel to a current-carrying wire at rest in the laboratory system $S$. Let $I$ be the current flowing in the wire. In the $S$-frame, charge $q$ feels only the magnetic (Lorentz) force

$$
\mathbf{F}=q \mathbf{v}_{\mathbf{0}} \times \mathbf{B} .
$$

Using Ampère's law, the magnetic field generated by a current $I$ at distance $r$ from the current-carrying wire is $B=\frac{1}{4 \pi \epsilon_{0} c^{2}} \frac{2 I}{r}$, where $\epsilon_{0}$ is the vacuum permittivity and $c$ is the speed of light in vacuum, and then the magnitude of the force felts by the charge $q$ is

$$
F=\frac{1}{4 \pi \epsilon_{0} c^{2}} \frac{2\left|I q v_{0}\right|}{r} .
$$

The force is repulsive if $q<0$ and $v_{0}$ is in the same direction as $I$, or if $q>0$ and $v_{0}$ is in the opposite direction of $I$.

In the typical atomic description of a metal wire carrying a current, the electric current comes from the motion of some of the negative electrons (conduction electrons) while the positive charges of the metal stay fixed. Let the density of the conduction electrons be $n_{-}(<0)$ and their velocity in $S$ be $v$. The density of the positive charges at rest in $S$ is $n_{+}$, which is always considered to be equal to the negative of $n_{-}$since we are considering an originally neutral wire and the wire remains uncharged also when the current passes through it.

Thus, the current $I$ can be written as $n_{-} v A$, where $A$ is the area of the cross-section of the wire (notice that the direction of the current $I$ is opposite to the velocity of the conduction electrons). Then

$$
F=\frac{1}{4 \pi \epsilon_{0} c^{2}} \frac{2\left|q n_{-} A v v_{0}\right|}{r} .
$$

Without loss of generality, we take the velocity $v_{0}$ of the charge $q$ equal to the velocity $v$ of the conduction electrons, same direction and magnitude. Thus, with $v_{0}=v$ eq. (3) becomes

$$
F=\frac{|q|}{2 \pi \epsilon_{0}} \frac{\left|n_{-}\right| A}{r} \frac{v^{2}}{c^{2}}
$$

If $q>0$ then $F$ is repulsive, while if $q<0$ then $F$ is attractive.

Now let turn our attention to what happens in the reference frame $S^{\prime}$, in which the particle is at rest and the wire is running past with velocity $-v$ (the frame $S^{\prime}$ moves in the same direction as the conduction electrons and with the same speed). The particle is now at rest, so there is no magnetic force on it. However, there must be a force and Purcell's explanation comes into play. 
If we take a length $L_{0}$ of the wire (with cross-section $A_{0}$ ), in which there is a charge density $n_{0}$ of stationary charges, it will contain a total charge of $Q=n_{0} L_{0} A$. If the same charges are observed in a different frame to be moving with velocity $v$, they will be all contained in a piece of the material with the shorter (Lorentz contracted) length

$$
L=L_{0} \sqrt{1-v^{2} / c^{2}}
$$

but with the same area $A_{0}$ (since, according to special relativity, dimensions perpendicular to the motion are unchanged). If we call $n$ the density of charges in the frame in which they are moving, the total charge $Q$ will be $n L A_{0}$. This must be also equal to $n_{0} L_{0} A_{0}$ since the charge is frame invariant, so that $n L=n_{0} L_{0}$ or, from eq. (5),

$$
n=\frac{n_{0}}{\sqrt{1-v^{2} / c^{2}}} \text {. }
$$

We can use this result for the positive charge density $n_{+}$of our wire. In the frame $S$, these charges are at rest. In $S^{\prime}$, however, where the wire moves with velocity $-v$, the positive charge density becomes

$$
n_{+}^{\prime}=\frac{n_{+}}{\sqrt{1-v^{2} / c^{2}}} .
$$

The negative charges, on the other hand, are at rest in $S^{\prime}$. Their rest density is $n_{-}^{\prime}$ and then

$$
n_{-}=\frac{n_{-}^{\prime}}{\sqrt{1-v^{2} / c^{2}}}, \quad \text { or } \quad n_{-}^{\prime}=n_{-} \sqrt{1-v^{2} / c^{2}} .
$$

We now see that, according to these calculations, there is an electric field in $S^{\prime}$ because in this frame the net charge density $n^{\prime}$ is equal to

$$
n^{\prime}=n_{+}^{\prime}+n_{-}^{\prime}=\frac{n_{+}}{\sqrt{1-v^{2} / c^{2}}}+n_{-} \sqrt{1-v^{2} / c^{2}}=n_{+} \frac{v^{2} / c^{2}}{\sqrt{1-v^{2} / c^{2}}}>0,
$$

since in $S$ the wire is neutral and $n_{-}=-n_{+}$.

The moving wire is thus positively charged and will produce an electric field $E^{\prime}$. This field is the same generated by a stationary wire uniformly charged with linear charge density $\lambda^{\prime}=n^{\prime} A$. The electric field at the distance $r$ from the axis of the wire is

$$
E^{\prime}=\frac{\lambda^{\prime}}{2 \pi \epsilon_{0} r}=\frac{n^{\prime} A}{2 \pi \epsilon_{0} r}=\frac{n_{+} A v^{2} / c^{2}}{2 \pi \epsilon_{0} r \sqrt{1-v^{2} / c^{2}}} .
$$

The electrostatic force acting upon the charge $q$ at rest in $S^{\prime}$ is then

$$
F^{\prime}=q E^{\prime}=\frac{q}{2 \pi \epsilon_{0}} \frac{n_{+} A}{r} \frac{v^{2} / c^{2}}{\sqrt{1-v^{2} / c^{2}}} .
$$

Since we have taken the velocity of the frame $S^{\prime}$ equal to the (drift) velocity $v$ of the conduction electrons and since $v \ll c$ in ordinary current-carrying wires, equation (11) can be approximated to

$$
F^{\prime}=\frac{q}{2 \pi \epsilon_{0}} \frac{n_{+} A}{r} \frac{v^{2} / c^{2}}{\sqrt{1-v^{2} / c^{2}}} \simeq \frac{q}{2 \pi \epsilon_{0}} \frac{\left|n_{-}\right| A}{r} \frac{v^{2}}{c^{2}}
$$


which is exactly the magnetic force calculated in $S$ (eq. (4)) because of $n_{+}=\left|n_{-}\right|$.

If we take into account the relativity fact that forces also Lorentz transform when we go from one system the other, it can be found that the two ways of looking at the phenomenon do give the same physical result for any velocity of the particle.

Purcell thus succeeded in showing why the magnetic (Lorentz) force experienced by a charge moving parallel to a current-carrying wire is a purely electrostatic force in the rest frame of the charge: this force can be "mechanically" explained with relativistic length contraction and the consequent charge density modification in the wire. No abstract Lorentz transformations of fields are involved in the derivation of the result.

This explanation appears to be one of the most striking successes of special relativity: the calculations fit perfectly with the Lorentz force law and this seems to be one of the few relativity consequences affecting physical phenomena in everyday life.

\section{Some inconsistencies deriving from Purcell's explanation}

There are a few aspects of Purcell's explanation that appear to be problematic. Inside a metal wire at rest in the laboratory and passed through by a current, only conduction electrons move (drift). To an observer in the laboratory, their density should appear augmented with respect to the density of the stationary lattice positive charges (which is the same density of the electrons when the current is off) by a suitable Lorentz factor. Instead, in every quantitative treatment of the problem we have seen, even when the current is on, in the frame of the wire the charge density of the moving electrons is considered to be equal to the charge density of the stationary lattice positive charges $\left(n_{+}=\left|n_{-}\right|\right)$and, to all accounts, the wire is consistently treated as to be neutral in the laboratory frame.

Unless we are ready to accept that no Lorentz contraction takes place on the moving electrons with respect to the laboratory frame, we have to explain this behavior as though, in the laboratory frame, the electrons with a Lorentz augmented density rearrange themselves in order to restore the zero net charge that was on the wire before the current was on, more or less according to the same principle by which negative charges, separated from the positive ones over a metallic surface, move to cancel out the overall electric potential difference.

But then the question is: why do electrons not do the same to cancel out the overall electric potential difference (charge unbalance) also in the reference frame of the charge moving parallel to the wire? We see in all this a lack of reciprocity, the same reciprocity demanded by the principle of relativity. If the relativistic excess of positive charge on the wire, when seen by the moving charge, is real enough to attract that charge to

the wire, then this same excess of positive charge should be real enough (again, when seen in the reference frame of the moving charge) to attract more electrons along the wire from the device that generates the current, in order to reestablish the zero net charge that was there before the current was on [6]. Why does it not happen? The only possibility is that the wire and the current generator, when seen from the reference 
frame of the moving charge, appear overall positively charged (non-zero net charge in the whole system). However, this generates a paradox that shall be discussed more extensively in Section 3 .

There are few doubts that Purcell's relativistic explanation is quite impressive. However, can this same relativistic approach explain Ampère's force law between, for instance, two parallel wires passed through by currents in opposite directions? Purcell gives an affirmative answer in that regard. See, for instance, the Example and Fig. 5.23 on pages 263-264 in [1], where Purcell shows how his derivation "explains the mutual repulsion of conductors carrying currents in opposite directions".

We believe that Purcell's approach probably explains why an electron moving in one of the wires sees a repulsive force exerted by the other wire. If we consider, for a moment, what an external observer at rest in the same reference frame of the conduction electrons sees (the observer moves at the same velocity of the conduction electrons), things become more intricate in the two-wire case. According to this observer, the wires result overall oppositely charged. We simply apply the contraction argument to both wires: in the reference frame of the conduction electrons of a wire, that wire appears overall positively charged, while the other wire, where conduction electrons are seen to be moving faster than the associated positive charges, appears overall negatively charged. Then, they should attract one another, exactly the contrary of what Ampère's force law and experience tell us. The same discrepancy occurs in the case of two parallel wires carrying currents in the same direction: from Ampère's force law and laboratory experience we expect attraction, while Purcell's approach predicts that in the reference frame of the moving electrons both wires become overall positively charged and then they should repel each other.

If to explain what happens between two current-carrying wires we need to resort anyway to Ampère's force law, then the relativistic explanation of the magnetic force made by Purcell is not general and thus it cannot be a basic, fundamental explanation of magnetic forces. Ampère's force is a magnetic force (closely related to the Lorentz force law) and Purcell's approach cannot explain it with the sole relativistic modification of the densities of the conduction electrons and the fixed positive charges, as has been done to successfully explain the Lorentz force law. The exclusive application of Purcell's relativistic explanation predicts the opposite result and we need always to resort to the Ampère force law to match the observations.

In the following section, we provide a further simple argument against the interpretation of Purcell's derivation as something that physically happens in the wire.

\section{Superconducting ring paradox}

Consider a superconducting ring of radius $R$ in which a direct current $I$ circulates clockwise. In the reference frame of the laboratory, the ring is uncharged, $Q_{t o t}=0$. The ring, when properly cooled, is able to maintain the current with no applied voltage 


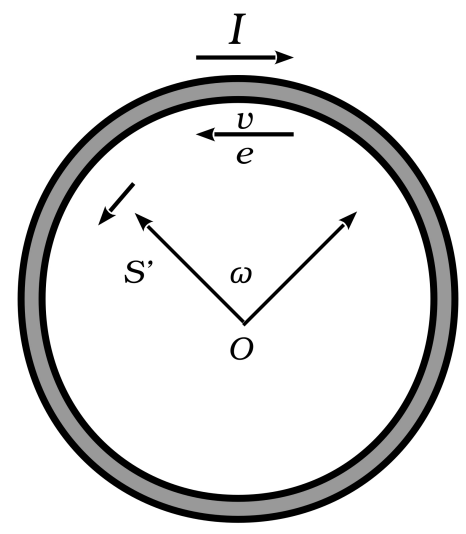

Figure 1. Superconducting ring and rotating reference frame $S^{\prime}$.

for years without any measurable degradation用 The current generates a magnetic field that can be very intense. The current $I$ is the result of conduction electrons moving counterclockwise.

Consider further a reference frame $S^{\prime}$ with origin $O$ that coincides with the center of the ring, and rotating counterclockwise with an angular velocity $\omega=v / R$ with $v \ll c$ (Fig. 11). An observer at rest in $S^{\prime}$ will see the conduction electrons as slowed down and the lattice positive charges of the ring as moving clockwise with velocity $v$. The centripetal acceleration experienced by $S^{\prime}$ can me made so small that we can treat this example with the machinery of special relativity (for instance, for $v<1 \mathrm{~m} / \mathrm{s}$ and $R \gtrsim 1 \mathrm{~m} a=\frac{v^{2}}{R}<1 \mathrm{~m} / \mathrm{s}^{2}$ ). General relativity becomes special relativity at the limit of a weak field.

If we apply Purcell's approach to the current in the ring, the observer in $S^{\prime}$ will detect on the whole ring a net positive charge density equal to that obtained in Section 1 . eq. (9),

$$
n^{\prime}=n_{+} \frac{v^{2} / c^{2}}{\sqrt{1-v^{2} / c^{2}}}
$$

Here, in some sense, the observer is taking the place of the test particle described in Section 1 ,

If we multiply $n^{\prime}$ by the volume of the ring, we notice that in $S^{\prime}$ the ring is not uncharged, $Q_{\text {tot }}^{\prime} \neq 0$ s.

This result is at odds with the universal principle of charge conservation in isolated systems. The total charge of the ring must be the same in both reference frames. What we have just shown represents also a corroboration of the heuristic observations made in

$\ddagger$ The fact that the ring is made of superconducting material is not essential for the derivation of our main result. One may also consider a ring made from metal with extremely low ohmic resistance. In this case, the current will last only for a few seconds.

$\S$ The conclusion does not change if in $S^{\prime}$ we consider a Lorentz contracted ring volume. 
the first part of Section 2 about the electrical neutrality of a rectilinear wire in different reference frames.

For those who still complain that $S^{\prime}$ is not an inertial frame and thus special relativity cannot be applied, consider the following variant. The reference frame $S^{\prime}$ is now at rest and the ring is set in rotation clockwise with angular velocity $\omega=v / R$. To an observer at rest in $S^{\prime}$, the conduction electrons in the ring appear as slowed down, while the lattice positive charges move clockwise with velocity $v$. By applying the contraction argument, this time from an inertial reference frame, the ring appears overall positively charged to the observer in $S^{\prime}$. This is again in contradiction with the universal principle of charge conservation in isolated systems. It is not possible to charge a neutral current-carrying ring by simply setting it into rotation.

In physics, it is well known that one can generate a potential difference between the center and the edge of a neutral metallic disk by simply setting the disk into rotation, but the overall charge of the disk remains always zero in this case.

\section{Conclusions}

We have shown that it is not possible to completely (and exclusively) explain the magnetic force experienced by a charge moving in a static magnetic field with relativistic length contraction, as has been proposed by E.M. Purcell in the case of a field generated by a current-carrying wire. His explanation results to be at odds with basic physics principles like the conservation of total charge in isolated systems and even the principle of relativity.

We maintain that, despite its suggestive agreement with the phenomenological laws of magnetic forces (the Lorentz force law but not the Ampère force law), Purcell's basic explanation has only an illustrative and expository value and cannot be considered as a real physical theory describing what happens in a current-carrying wire. We believe that the difficulties pointed out here should be explicitly presented and discussed when

introducing Purcell's approach in physics courses at the undergraduate and graduate levels.

Electric and magnetic fields are interrelated, and this fact is implicit in Maxwell's equations, but Purcell's relativistic explanation of this interrelatedness does not work. The Lorentz transformations of the electric and magnetic fields already tell that a magnetic field Lorentz transforms into an electric field (and, by necessity, also into a magnetic field since a purely magnetic field cannot be transformed into a purely electric field). Here, though, we show that the electric field coming from the Lorentz transformation of the purely magnetic field generated by a current-carrying wire cannot be simply and "mechanically" explained with charge density unbalance in the wire due to relativistic length contraction.

Our conclusions, although coming from an analysis limited to the very specific approach of Purcell, agree with the allegedly more general result obtained by Jefimenko [5] according to which neither the magnetic nor the electric field is a 
relativistic effect.

\section{Acknowledgments}

The author is indebted to Dr. Assunta Tataranni and Dr. Gianpietro Summa for key improvements of the manuscript. The author acknowledges anonymous reviewers for their valuable comments and suggestions.

\section{References}

[1] Purcell E M and Morin D J 2013 Electricity and Magnetism Harvard University Mass. Third edition pp 259-264. First edition published by Education Development Center Inc. in 1963

[2] Born M 1964 Die Relativitätstheorie Einstein Springer-Verlag Berlin Heidelberg GmbH pp 253-256

[3] Feynman R P, Leighton R B and Sands M 1964 The Feynman Lectures on Physics Addison-Wesley, Reading Mass. Vol. 2, Section 13-6

[4] Sexl R and Schmidt H K 1978 Raum - Zeit - Relativität Rowohlt Taschenbuch Verlag Hamburg GmbH pp 168-175

[5] Jefimenko O D 1996 Is magnetic field due to an electric current a relativistic effect? Eur. J. Phys. 17 180-182

[6] D'Abramo G 2017 Probing the Limits: Collected Works on the Second Law of Thermodynamics and Special Relativity. CreateSpace Independent Publishing Platform. ISBN: 978-1540844781 pp 124-126 